\title{
Managing Common Interventional Radiology Complications
}



Lakshmi Ratnam • Uday Patel Anna Maria Belli

Editors

\section{Managing Common Interventional Radiology Complications}

A Case Based Approach

Springer 
Editors

Lakshmi Ratnam

Department of Radiology

St. George's Hospital

London

UK

Uday Patel

Department of Radiology

St. George's Hospital

and Medical School

London

UK
Anna Maria Belli

Department of Radiology

St. George's Hospital

London

UK

ISBN 978-1-4471-5501-0

DOI 10.1007/978-1-4471-5502-7

ISBN 978-1-4471-5502-7 (eBook)

Springer London Heidelberg New York Dordrecht

Library of Congress Control Number: 2013954803

(C) Springer-Verlag London 2014

This work is subject to copyright. All rights are reserved by the Publisher, whether the whole or part of the material is concerned, specifically the rights of translation, reprinting, reuse of illustrations, recitation, broadcasting, reproduction on microfilms or in any other physical way, and transmission or information storage and retrieval, electronic adaptation, computer software, or by similar or dissimilar methodology now known or hereafter developed. Exempted from this legal reservation are brief excerpts in connection with reviews or scholarly analysis or material supplied specifically for the purpose of being entered and executed on a computer system, for exclusive use by the purchaser of the work. Duplication of this publication or parts thereof is permitted only under the provisions of the Copyright Law of the Publisher's location, in its current version, and permission for use must always be obtained from Springer. Permissions for use may be obtained through RightsLink at the Copyright Clearance Center. Violations are liable to prosecution under the respective Copyright Law.

The use of general descriptive names, registered names, trademarks, service marks, etc. in this publication does not imply, even in the absence of a specific statement, that such names are exempt from the relevant protective laws and regulations and therefore free for general use.

While the advice and information in this book are believed to be true and accurate at the date of publication, neither the authors nor the editors nor the publisher can accept any legal responsibility for any errors or omissions that may be made. The publisher makes no warranty, express or implied, with respect to the material contained herein.

Printed on acid-free paper

Springer is part of Springer Science+Business Media (www.springer.com) 
To all our teachers 



\section{Preface}

Although among the younger medical specialities, interventional radiology has rapidly established a central position in modern medical practice. The number and range of IR procedures is now wide and new procedures are pioneered all the time.

Whether of a minor nature - say, image guided biopsy or drainage - or complex, such as superselective arterial embolization and EVAR, IR techniques have a high success rate, but more importantly being minimally invasive the morbidity is more favourable compared to alternative procedures, be they surgical or medical. Nevertheless, complications can and do occur, and it is the duty of every IR practitioner to not only understand how and why such complications occur, so that they can be avoided, but also be technically armed to rectify the complication as speedily and safely as possible. Although any complication is in its way unique, there are always common themes.

An anonymized format has been adopted, by listing all the contributors to this book jointly at the head of each chapter describing a real complication. The practitioners who dealt with the complication describe how they managed the problem and the eventual outcome. Each chapter also discusses how such problems arise and the principles behind their early recognition and eventual management. Some general tips are also given for the benefit of those who have not yet encountered such a complication. 
viii Preface

Finally, a general commentary is provided, written by the editors, which is a reflection on the problem and describes, when applicable, alternative strategies to the complication.

London, UK

Dr. Lakshmi Ratnam

London, UK

Dr. Uday Patel

London, UK

Prof. Anna Maria Belli 


\section{Acknowledgements}

The editors are deeply grateful to all the authors who shared their clinical experience, without whom this book would have not been feasible. The help and encouragement of our IR colleagues and staff is also gratefully acknowledged. Finally, we would like to say thank you to our family and friends for always being there. 



\section{Contents}

\section{Embolization Coil Trapped in the Side}

Hole of a Catheter...................... 1

Robert P. Allison, Anna Maria Belli,

Joo-Young Chun, Raymond Chung, Raj Das,

Andrew England, Karen Flood,

Marie-France Giroux, Richard G. McWilliams,

Robert Morgan, Nik Papadakos, Jai V. Patel,

Raf Patel, Uday Patel, Lakshmi Ratnam,

Reddi Prasad Yadavali, and John Rose

\section{Retrieval of an Intravascular Foreign}

Body in the Venous System ................. 7

Robert P. Allison, Anna Maria Belli, Joo-Young Chun, Raymond Chung, Raj Das, Andrew England, Karen Flood,

Marie-France Giroux, Richard G. McWilliams,

Robert Morgan, Nik Papadakos, Jai V. Patel,

Raf Patel, Uday Patel, Lakshmi Ratnam,

Reddi Prasad Yadavali, and John Rose

\section{Migrated Superior Vena Cava Stent}

Repositioned Using a Balloon

and Loop Snare Combination ................ 13

Robert P. Allison, Anna Maria Belli,

Joo-Young Chun, Raymond Chung, Raj Das,

Andrew England, Karen Flood,

Marie-France Giroux, Richard G. McWilliams,

Robert Morgan, Nik Papadakos, Jai V. Patel,

Raf Patel, Uday Patel, Lakshmi Ratnam,

Reddi Prasad Yadavali, and John Rose 


\section{Retrieval of Central Venous Catheter Fragment}

Following Portacath Removal . . . . . . . . . . . . 21

Robert P. Allison, Anna Maria Belli, Joo-Young Chun, Raymond Chung, Raj Das, Andrew England, Karen Flood,

Marie-France Giroux, Richard G. McWilliams, Robert Morgan, Nik Papadakos, Jai V. Patel, Raf Patel, Uday Patel, Lakshmi Ratnam, Reddi Prasad Yadavali, and John Rose

\section{Central Venous Catheter Inserted} into the Mediastinum . . . . . . . . . . . . . . . . . 29

Robert P. Allison, Anna Maria Belli, Joo-Young Chun, Raymond Chung, Raj Das, Andrew England, Karen Flood, Marie-France Giroux, Richard G. McWilliams, Robert Morgan, Nik Papadakos, Jai V. Patel, Raf Patel, Uday Patel, Lakshmi Ratnam, Reddi Prasad Yadavali, and John Rose

\section{Femoral Artery Pseudoaneurysm Treated} with Percutaneous Thrombin Injection

Robert P. Allison, Anna Maria Belli, Joo-Young Chun, Raymond Chung, Raj Das, Andrew England, Karen Flood, Marie-France Giroux, Richard G. McWilliams, Robert Morgan, Nik Papadakos, Jai V. Patel, Raf Patel, Uday Patel, Lakshmi Ratnam, Reddi Prasad Yadavali, and John Rose

\section{Superficial Femoral Artery Thrombosis}

Post-angioplasty . . . . . . . . . . . . . . . . 45

Robert P. Allison, Anna Maria Belli, Joo-Young Chun, Raymond Chung, Raj Das, Andrew England, Karen Flood, Marie-France Giroux, Richard G. McWilliams, Robert Morgan, Nik Papadakos, Jai V. Patel, Raf Patel, Uday Patel, Lakshmi Ratnam, Reddi Prasad Yadavali, and John Rose 


\section{Superficial Femoral}

Artery Rupture Following Angioplasty . . . . . . . . 55

Robert P. Allison, Anna Maria Belli, Joo-Young Chun, Raymond Chung, Raj Das, Andrew England, Karen Flood,

Marie-France Giroux, Richard G. McWilliams, Robert Morgan, Nik Papadakos, Jai V. Patel, Raf Patel, Uday Patel, Lakshmi Ratnam, Reddi Prasad Yadavali, and John Rose

\section{Distal Embolization Following Common Iliac} and Superficial Femoral Artery Angioplasty .....

Robert P. Allison, Anna Maria Belli, Joo-Young Chun, Raymond Chung, Raj Das, Andrew England, Karen Flood,

Marie-France Giroux, Richard G. McWilliams, Robert Morgan, Nik Papadakos, Jai V. Patel, Raf Patel, Uday Patel, Lakshmi Ratnam, Reddi Prasad Yadavali, and John Rose

\section{Flow-Limiting Iliac Artery Dissection} Post-angioplasty . . . . . . . . . . . . . . .

Robert P. Allison, Anna Maria Belli, Joo-Young Chun, Raymond Chung, Raj Das, Andrew England, Karen Flood,

Marie-France Giroux, Richard G. McWilliams, Robert Morgan, Nik Papadakos, Jai V. Patel, Raf Patel, Uday Patel, Lakshmi Ratnam, Reddi Prasad Yadavali, and John Rose

11 Dissection of Superior Mesenteric Artery (SMA) During Transarterial Chemoembolization (TACE) via a Replaced Right Hepatic Artery . . . .

Robert P. Allison, Anna Maria Belli, Joo-Young Chun, Raymond Chung, Raj Das, Andrew England, Karen Flood,

Marie-France Giroux, Richard G. McWilliams, Robert Morgan, Nik Papadakos, Jai V. Patel, Raf Patel, Uday Patel, Lakshmi Ratnam, Reddi Prasad Yadavali, and John Rose 
12 Iatrogenic Iliac Artery Rupture During Arterial Stenting

Robert P. Allison, Anna Maria Belli, Joo-Young Chun, Raymond Chung, Raj Das, Andrew England, Karen Flood,

Marie-France Giroux, Richard G. McWilliams, Robert Morgan, Nik Papadakos, Jai V. Patel, Raf Patel, Uday Patel, Lakshmi Ratnam, Reddi Prasad Yadavali, and John Rose

\section{Detachment of a Balloon-Expandable}

Stent from the Balloon and the Wire . . . . . . . . . 89

Robert P. Allison, Anna Maria Belli, Joo-Young Chun, Raymond Chung, Raj Das, Andrew England, Karen Flood, Marie-France Giroux, Richard G. McWilliams, Robert Morgan, Nik Papadakos, Jai V. Patel, Raf Patel, Uday Patel, Lakshmi Ratnam, Reddi Prasad Yadavali, and John Rose

\section{Migration of Common Hepatic Artery Stent} Graft Occluding Right Hepatic Artery Flow . . . . . .

Robert P. Allison, Anna Maria Belli, Joo-Young Chun, Raymond Chung, Raj Das, Andrew England, Karen Flood,

Marie-France Giroux, Richard G. McWilliams, Robert Morgan, Nik Papadakos, Jai V. Patel, Raf Patel, Uday Patel, Lakshmi Ratnam, Reddi Prasad Yadavali, and John Rose

15 Migrated Stent Graft During TIPS Revision..... . 105 Robert P. Allison, Anna Maria Belli, Joo-Young Chun, Raymond Chung, Raj Das, Andrew England, Karen Flood, Marie-France Giroux, Richard G. McWilliams, Robert Morgan, Nik Papadakos, Jai V. Patel, Raf Patel, Uday Patel, Lakshmi Ratnam, Reddi Prasad Yadavali, and John Rose 


\section{Type 1A Endoleak Following EVAR}

Treated with a Proximal Cuff. . . . . . . . . . . . . . 113

Robert P. Allison, Anna Maria Belli, Joo-Young Chun, Raymond Chung, Raj Das, Andrew England, Karen Flood,

Marie-France Giroux, Richard G. McWilliams, Robert Morgan, Nik Papadakos, Jai V. Patel, Raf Patel, Uday Patel, Lakshmi Ratnam, Reddi Prasad Yadavali, and John Rose

\section{Management of a Type 1B Endoleak}

\section{Following EVAR}

Robert P. Allison, Anna Maria Belli, Joo-Young Chun, Raymond Chung, Raj Das, Andrew England, Karen Flood, Marie-France Giroux, Richard G. McWilliams, Robert Morgan, Nik Papadakos, Jai V. Patel, Raf Patel, Uday Patel, Lakshmi Ratnam, Reddi Prasad Yadavali, and John Rose

\section{Persistent Type 2 Endoleak Post-EVAR} with Aneurysm Expansion

Robert P. Allison, Anna Maria Belli, Joo-Young Chun, Raymond Chung, Raj Das, Andrew England, Karen Flood,

Marie-France Giroux, Richard G. McWilliams, Robert Morgan, Nik Papadakos, Jai V. Patel, Raf Patel, Uday Patel, Lakshmi Ratnam, Reddi Prasad Yadavali, and John Rose

19 Acute Renal Artery Occlusion and Trapped Renal Artery Catheter During Infrarenal AAA Stent Grafting

Robert P. Allison, Anna Maria Belli, Joo-Young Chun, Raymond Chung, Raj Das, Andrew England, Karen Flood,

Marie-France Giroux, Richard G. McWilliams, Robert Morgan, Nik Papadakos, Jai V. Patel, Raf Patel, Uday Patel, Lakshmi Ratnam, Reddi Prasad Yadavali, and John Rose 
20 Maldeployment of the Contralateral

Limb During EVAR . . . . . . . . . . . . . . . . . . . . . . 149

Robert P. Allison, Anna Maria Belli, Joo-Young Chun, Raymond Chung, Raj Das, Andrew England, Karen Flood,

Marie-France Giroux, Richard G. McWilliams, Robert Morgan, Nik Papadakos, Jai V. Patel, Raf Patel, Uday Patel, Lakshmi Ratnam, Reddi Prasad Yadavali, and John Rose

\section{Branch Endograft Disconnection}

and Impending Type 3 Endoleak Post-EVAR. . . . 157

Robert P. Allison, Anna Maria Belli, Joo-Young Chun, Raymond Chung, Raj Das, Andrew England, Karen Flood, Marie-France Giroux, Richard G. McWilliams, Robert Morgan, Nik Papadakos, Jai V. Patel, Raf Patel, Uday Patel, Lakshmi Ratnam, Reddi Prasad Yadavali, and John Rose

\section{Hemorrhage Following Percutaneous}

Nephrostomy . . . . . . . . . . . . . . . . . . 167

Robert P. Allison, Anna Maria Belli, Joo-Young Chun, Raymond Chung, Raj Das, Andrew England, Karen Flood,

Marie-France Giroux, Richard G. McWilliams, Robert Morgan, Nik Papadakos, Jai V. Patel, Raf Patel, Uday Patel, Lakshmi Ratnam, Reddi Prasad Yadavali, and John Rose

\section{Injury to Bowel Following Transplant}

Nephrostomy Insertion . . . . . . . . . . . . . . . . . 175

Robert P. Allison, Anna Maria Belli, Joo-Young Chun, Raymond Chung, Raj Das, Andrew England, Karen Flood, Marie-France Giroux, Richard G. McWilliams, Robert Morgan, Nik Papadakos, Jai V. Patel, Raf Patel, Uday Patel, Lakshmi Ratnam, Reddi Prasad Yadavali, and John Rose 


\section{Renal Arterial Hemorrhage Following}

Renal Artery Stenting . . . . . . . . . . . . . . . 183

Robert P. Allison, Anna Maria Belli, Joo-Young Chun, Raymond Chung, Raj Das, Andrew England, Karen Flood,

Marie-France Giroux, Richard G. McWilliams, Robert Morgan, Nik Papadakos, Jai V. Patel, Raf Patel, Uday Patel, Lakshmi Ratnam, Reddi Prasad Yadavali, and John Rose

\section{Pyrexia After Tumor Embolization: Infection} Versus Post-embolization Syndrome. . . . . . . . . . . . 191

Robert P. Allison, Anna Maria Belli, Joo-Young Chun, Raymond Chung, Raj Das, Andrew England, Karen Flood, Marie-France Giroux, Richard G. McWilliams, Robert Morgan, Nik Papadakos, Jai V. Patel, Raf Patel, Uday Patel, Lakshmi Ratnam, Reddi Prasad Yadavali, and John Rose

26 Arterioportal Fistula and Liver Hemorrhage After Radiofrequency Ablation and TACE . . . . . . 199 Robert P. Allison, Anna Maria Belli, Joo-Young Chun, Raymond Chung, Raj Das, Andrew England, Karen Flood, Marie-France Giroux, Richard G. McWilliams, Robert Morgan, Nik Papadakos, Jai V. Patel, Raf Patel, Uday Patel, Lakshmi Ratnam, Reddi Prasad Yadavali, and John Rose

\section{Protrusion of Vena Cava Filter} into the Aorta . . . . . . . . . . . . . . . . . . . . . . . 205

Robert P. Allison, Anna Maria Belli, Joo-Young Chun, Raymond Chung, Raj Das, Andrew England, Karen Flood, Marie-France Giroux, Richard G. McWilliams, Robert Morgan, Nik Papadakos, Jai V. Patel, Raf Patel, Uday Patel, Lakshmi Ratnam, Reddi Prasad Yadavali, and John Rose 


\section{The Multiple Options}

for Retrieval of a Tilted IVC Filter . . . . . . . . . . . 213

Robert P. Allison, Anna Maria Belli, Joo-Young Chun, Raymond Chung, Raj Das, Andrew England, Karen Flood,

Marie-France Giroux, Richard G. McWilliams,

Robert Morgan, Nik Papadakos, Jai V. Patel, Raf Patel, Uday Patel, Lakshmi Ratnam, Reddi Prasad Yadavali, and John Rose

\section{Retrieval of a Well-Orientated IVC} Filter with Embedded Struts . . . . . . . . . . . . . . . . . 221

Robert P. Allison, Anna Maria Belli, Joo-Young Chun, Raymond Chung, Raj Das, Andrew England, Karen Flood,

Marie-France Giroux, Richard G. McWilliams, Robert Morgan, Nik Papadakos, Jai V. Patel, Raf Patel, Uday Patel, Lakshmi Ratnam, Reddi Prasad Yadavali, and John Rose

\section{Retrieving a Tilted IVC}

Filter with Struts Penetrating the IVC . . . . . . . . . 227

Robert P. Allison, Anna Maria Belli, Joo-Young Chun, Raymond Chung, Raj Das, Andrew England, Karen Flood,

Marie-France Giroux, Richard G. McWilliams, Robert Morgan, Nik Papadakos, Jai V. Patel, Raf Patel, Uday Patel, Lakshmi Ratnam, Reddi Prasad Yadavali, and John Rose

31 Fistula Rupture Post-fistuloplasty . . . . . . . . . . . 235

Robert P. Allison, Anna Maria Belli, Joo-Young Chun, Raymond Chung, Raj Das, Andrew England, Karen Flood, Marie-France Giroux, Richard G. McWilliams, Robert Morgan, Nik Papadakos, Jai V. Patel, Raf Patel, Uday Patel, Lakshmi Ratnam, Reddi Prasad Yadavali, and John Rose 
32 Circumferential Balloon Rupture and Retained Fragments During Fistuloplasty and Thrombolysis of a Thrombosed Fistula . . . . . . 243 Robert P. Allison, Anna Maria Belli, Joo-Young Chun, Raymond Chung, Raj Das, Andrew England, Karen Flood, Marie-France Giroux, Richard G. McWilliams, Robert Morgan, Nik Papadakos, Jai V. Patel, Raf Patel, Uday Patel, Lakshmi Ratnam, Reddi Prasad Yadavali, and John Rose

\section{Hemorrhage After Transjugular}

Liver Biopsy . . . . . . . . . . . . . . . . . . . . . . . . . 251

Robert P. Allison, Anna Maria Belli, Joo-Young Chun, Raymond Chung, Raj Das, Andrew England, Karen Flood,

Marie-France Giroux, Richard G. McWilliams, Robert Morgan, Nik Papadakos, Jai V. Patel, Raf Patel, Uday Patel, Lakshmi Ratnam, Reddi Prasad Yadavali, and John Rose 



\section{Contributors}

Robert P. Allison Department of Interventional Radiology, University Hospitals Southampton, Southampton, UK

Anna Maria Belli Department of Radiology, St. George's Hospital and Medical School, London, UK Joo-Young Chun Department of Radiology, St. George's Hospital, London, UK

Raymond Chung Department of Radiology, St. George's Hospital, London, UK

Raj Das Department of Radiology, St. George's Hospital, London, UK

Andrew England Department of Radiography, University of Salford, Manchester, UK

Karen Flood Department of Vascular Radiology, Leeds General Infirmary, Leeds, UK

Marie-France Giroux Department of Radiology, CHUM-Centre Hospitalier de l'Université de Montréal, Montreal, QC, Canada

Richard G. McWilliams Department of Radiology, Royal Liverpool University Hospital, Liverpool, UK

Robert Morgan Department of Radiology,

St. George's Hospital, London, UK

Nik Papadakos Department of Radiology,

St. George's Hospital, London, UK 
Jai V. Patel Department of Radiology, The Leeds Teaching Hospitals NHS Trust, Leeds, UK

Raf Patel Department of Radiology, The Leeds Teaching Hospitals NHS Trust, Leeds, West Yorkshire, UK

Uday Patel Department of Radiology, St. George's Hospital and Medical School, London, UK

Lakshmi Ratnam Department of Radiology, St. George's Hospital, London, UK

John Rose Department of Interventional Radiology, Freeman Hospital, Newcastle Upon Tyne Hospitals NHS Trust, Newcastle upon Tyne, UK

Reddi Prasad Yadavali Department of Radiology, Aberdeen Royal Infirmary, Aberdeen, UK 